February 24， 2013 15:12 WSPC/INSTRUCTION FILE RKaminskiMeson 2008

International Journal of Modern Physics A

(C) World Scientific Publishing Company

\title{
NEW DISPERSION RELATIONS IN THE DESCRIPTION OF $\pi \pi$ SCATTERING AMPLITUDES
}

\author{
R. Kamiński ${ }^{a, *}$, R. García-Martín ${ }^{b}$, P. Grynkiewicz ${ }^{a}$ J. R. Peláez ${ }^{b}$, F. J. Ynduráin ${ }^{c}$ \\ ${ }^{a}$ Department of Theoretical Physics, H. Niewodniczański Institute of Nuclear Physics, Polish \\ Academy of Sciences, 31-342 Kraków, Poland \\ ${ }^{b}$ Departamento de Física Teórica II (Métodos Matemáticos), Facultad de Ciencias Físicas, \\ Universidad Complutense de Madrid, E-28040, Madrid, Spain \\ ${ }^{c}$ Departamento de Física Teórica, C-XI Universidad Autónoma de Madrid, Canto Blanco, \\ E-28049, Madrid, Spain
}

\begin{abstract}
We present a set of once subtracted dispersion relations which implement crossing symmetry conditions for the $\pi \pi$ scattering amplitudes below $1 \mathrm{GeV}$. We compare and discuss the results obtained for the once and twice subtracted dispersion relations, known as Roy's equations, for three $\pi \pi$ partial $J I$ waves, $S 0, P$ and $S 2$. We also show that once subtracted dispersion relations provide a stringent test of crossing and analyticity for $\pi \pi$ partial wave amplitudes, remarkably precise in the 400 to $1.1 \mathrm{GeV}$ region, where the resulting uncertainties are significantly smaller than those coming from standard Roy's equations, given the same input.
\end{abstract}

Keywords: pion-pion amplitudes, Roy's equations

PACS numbers: 13.75.Lb, 11.55.-m, 11.55.Fv, 11.80.Et

\section{Introduction}

In 1971 S. M. Roy 1 derived a set of coupled integral equations, the Roy Equations (RE), for the $\pi \pi$ scattering partial waves, by implementing crossing symmetry conditions into twice subtracted dispersion relations. In recent years, Roy's equations have been used in several ways: to obtain predictions for low energy $\pi \pi$ scattering using Chiral Perturbation Theory $(\mathrm{ChPT}){ }^{2 / 3}$, to test those predictions $(\mathrm{ChPT})^{4 / 516}$, and also to eliminate the well known "up-down" ambiguity 718 .

In a series of works, our group $[5]$ has also used a dispersive approach, to obtain, using also the most recent experimental results, a precise data parametrization of $\pi \pi$ scattering amplitudes consistent with analyticity, unitarity and crossing. In fact, the recent data from E865 collaboration at Brookhaven 9 and from NA48/2 $\frac{10}{10}$ provide us with new and very precise information on the $\pi \pi$ scattering at low energies. In our works we have combined Forward Dispersion Relations (FDR) and Roy's Eqs. Let us remark that we have only used the very general properties of analyticity, crossing, etc... and data, so that the approach is model independent. Furthermore,

*E-mail address: Robert.Kaminski@ifj.edu.pl 
we have not included ChPT constraints, so that our results could be used as tests of ChPT. The advantage of FDR is that they are very precise, can be extended up to any energy and do not depend on the large $t$ behavior. In contrast, Roy Eqs. use the full $t$ dependence since they are written in terms of partial waves and can only be used up to roughly $1.1 \mathrm{GeV}$. However, RE provide a simple and well defined analytic extension of partial waves for the calculation of poles in the complex plane. Such analytic extension of $\pi \pi$ scattering partial waves to the complex plane is of particular interest for the understanding of the controversial sigma resonance. Actually, Roy's equations have been used to predict very precisely the sigma pole position 11 using the ChPT determination of the scattering lengths.

We report here about our work in progress to improve our description of the energy region above $400 \mathrm{MeV}$, that can subsequently provide a precise determination of the sigma pole. Actually, when using standard RE, the large experimental error of the scattering length $a_{0}^{2}$ of the isospin 2 scalar partial wave, becomes a very large uncertainty in the intermediate energy region and for the sigma pole determination. For this reason we briefly describe here a new set of once-subtracted RE, denoted GKPY Eqs. for brevity, and we show the relative sizes of the different contributions, comparing them with those for standard RE. We show that, given the same input, the uncertainties of standard Roy's Eqs. are smaller than those of GKPY Eqs. at low energies. However, the uncertainties of the once-subtracted GKPY Eqs. are smaller than those of Roy's Eqs. above, roughly $400 \mathrm{MeV}$, up to $1.1 \mathrm{GeV}$. Hence, in that energy region, GKPY provide a very precise additional constraint for our dispersive analysis of data, and a very precise analytic extension to determine the position of the sigma pole from experiment.

\section{Once and Twice Subtracted Dispersion Relations}

A twice subtracted dispersion relation for the scattering amplitude $T(s, t)$ of a given process is an expression of the form:

$$
\begin{aligned}
\operatorname{ReT}(s) & =g\left(s_{1}, s_{2}\right)+h\left(s ; s_{1}, s_{2}\right)+\frac{\left(s_{1}-s\right)\left(s_{2}-s\right)}{\pi} \int_{s_{t h}}^{\infty} \frac{\operatorname{Im} T(s \prime)}{\left(s^{\prime}-s\right)\left(s^{\prime}-s_{1}\right)\left(s^{\prime}-s_{2}\right)} d s \prime \\
& +\frac{\left(s_{1}-s\right)\left(s_{2}-s\right)}{\pi} \int_{-t}^{-\infty} \frac{\operatorname{ImT}\left(s^{\prime}\right)}{\left(s^{\prime}-s\right)\left(s^{\prime}-s_{1}\right)\left(s^{\prime}-s_{2}\right)} d s^{\prime}
\end{aligned}
$$

which relates the real part of an amplitude for a real $s$ value to the imaginary part of the amplitude integrated over the whole energy range, together with two functions, $g\left(t ; s_{1}, s_{2}\right)$ and $h\left(s, t ; s_{1}, s_{2}\right)$, called the subtraction terms, $S T(s)$. In the paper by Roy 1 such a relation is written for the definite $\pi \pi$ scattering isospin amplitudes, in a slightly modified way to show explicitly the crossing relations between the $s$ and $u$ channels, and in which the subtraction points are taken to be $s_{1}=s_{2}=0$. In addition, and for convenience, the three isospin amplitudes are written as an isospin vector amplitude $\vec{T}(s, t)=\left(T^{0}, T^{1}, T^{2}\right)$. This provides a relation among all 
the isospin processes by means of three crossing matrices $C_{s t}, C_{t u}, C_{s u}$, defined as:

$$
\vec{T}(s, t, u)=C_{s t} \vec{T}(t, s, u)=C_{s u} \vec{T}(u, t, s)=C_{t u} \vec{T}(s, u, t) .
$$

By using these, $s \leftrightarrow u$ crossing symmetry and the fact that on the $t$ channel the amplitudes with given isospin are of definite symmetry, the subtraction constants can be rewritten as $C_{s t}[\vec{C}(t)+(s-u) \vec{D}(t)]$, with $\vec{C}(t)=\left(c^{0}(t), 0, c^{1}(t)\right)$ and $\vec{D}(t)=$ $(0, d(t), 0)$. Thus,

$$
C_{s t} T\left(s=0, t=t_{0}, u=4 m_{\pi}^{2}-t_{0}\right)=T\left(s=t_{0}, t=0, u=4 m_{\pi}^{2}-t_{0}\right),
$$

which leads to:

$$
\begin{aligned}
C_{s t} T\left(0, t, 4 m_{\pi}^{2}-t\right) & =\frac{1}{\pi} \int_{4 m_{\pi}^{2}}^{\infty} d s \prime \frac{C_{s t} C_{s u} \operatorname{Im} \vec{T}(s \prime, t)}{s^{2}} \frac{\left(4 m_{\pi}^{2}-t\right)^{2}}{s^{\prime}-4 m_{\pi}^{2}+t} \\
& +\vec{C}(t)+\left(t-4 m_{\pi}^{2}\right) \vec{D}(t), \\
T\left(t, 0,4 m_{\pi}^{2}-t\right) & =\frac{1}{\pi} \int_{4 m_{\pi}^{2}}^{\infty} d s \prime\left(\frac{t^{2}}{s^{\prime}-t}+\frac{\left(4 m_{\pi}^{2}-t\right)^{2}}{s^{\prime}-4 m_{\pi}^{2}+t} C_{s u}\right) \frac{\operatorname{Im} \vec{T}(s \prime, 0)}{s^{2}} \\
& +C_{s t}\left[\vec{C}(0)+\left(2 t-4 m_{\pi}^{2}\right) \vec{D}(0)\right] .
\end{aligned}
$$

In order to express $\vec{C}(t)$ and $\vec{D}(t)$ in terms of known quantities one takes advantage of the fact that $\left(1 \pm C_{t u}\right) / 2$ are orthogonal projectors over the $s \leftrightarrow u$ symmetric or antisymmetric components, and evaluate the amplitude at threshold:

$\vec{T}\left(4 m_{\pi}^{2}, 0,0\right)=32 \pi\left(a_{0}^{0}, 0, a_{0}^{2}\right)=C_{s t}\left[\vec{C}(0)+4 m_{\pi}^{2} \vec{D}(0)\right]+\frac{1}{\pi} \int_{4 m_{\pi}^{2}}^{\infty} d s \prime \frac{\operatorname{Im} \vec{T}(s \prime, 0)}{s^{2}} \frac{16 m_{\pi}^{2}}{s \prime-4 m_{\pi}^{2}}$.

After projection into partial waves $T^{I}(s, t)=32 \pi \sum_{\ell}(2 \ell+1) P_{\ell}(x(t)) f_{\ell}^{I}(s)$ one obtains the full expression for Roy's equations:

$$
\begin{aligned}
\operatorname{Re} f_{\ell}^{I}(s) & =a_{0}^{0} \delta_{I 0} \delta_{\ell 0}+a_{0}^{2} \delta_{I 2} \delta_{\ell 0} \\
& +\frac{s-4 m_{\pi}^{2}}{12 m_{\pi}^{2}}\left(2 a_{0}^{0}-5 a_{0}^{2}\right)\left(\delta_{I 0} \delta_{\ell 0}+\frac{1}{6} \delta_{I 1} \delta_{\ell 1}-\frac{1}{2} \delta_{I 2} \delta_{\ell 0}\right) \\
& +\sum_{I^{\prime}=0}^{2} \sum_{\ell^{\prime}=0}^{1} f_{4 m_{\pi}^{2}}^{s_{\max }} d s^{\prime} K_{\ell \ell^{\prime}}^{I I^{\prime}}\left(s, s^{\prime}\right) \operatorname{Im} f_{\ell^{\prime}}^{I^{\prime}}\left(s^{\prime}\right)+d_{\ell}^{I}\left(s, s_{\max }\right)
\end{aligned}
$$

where the integrals with the kernels $K_{\ell \ell^{\prime}}^{I I^{\prime}}\left(s, s^{\prime}\right)$ contain the contributions of the $S 0, P$ and $S 2$ waves below $s_{\max }$, and are called kernel terms, $K T(s)$. The so called driving terms $d_{\ell}^{I}\left(s, s_{\max }\right)$ (abbreviated $D T(s)$ ) describe the influence of these waves above $s_{\max }$, and of the higher partial waves from the $\pi \pi$ threshold to infinity. In our previous analysis, the value $s_{\max }^{1 / 2}=1420 \mathrm{MeV}$ was chosen after studying the experimental data on the $\pi \pi$ scattering 6 . Above this energy a Regge parametrization is used. 
The derivation of the GKPY equations follows this very same pattern, but begins with a once subtracted dispersion relation. This leads to:

$$
\begin{aligned}
\operatorname{Re} f_{\ell}^{I}(s)= & \sum_{I^{\prime}} C_{I I^{\prime}}^{s t} a_{0}^{I^{\prime}}+\sum_{\ell^{\prime}}\left(2 \ell^{\prime}+1\right) \\
\times & \int_{4 m_{\pi}^{2}}^{s_{\max }^{2}} d s^{\prime}\left\{K_{\ell \ell^{\prime}}\left(s, s^{\prime}\right) \operatorname{Im} f_{\ell^{\prime}}^{I}\left(s^{\prime}\right)-L_{\ell \ell^{\prime}}\left(s, s^{\prime}\right) \sum_{I^{\prime}} C_{I I^{\prime}}^{s u} \operatorname{Im} f_{\ell^{\prime}}^{I^{\prime}}\left(s^{\prime}\right)\right. \\
& \left.+\sum_{I^{\prime \prime}} C_{I^{\prime \prime}}^{s t}\left[M_{\ell}\left(s, s^{\prime}\right) \operatorname{Im} f_{\ell^{\prime}}^{I^{\prime \prime}}\left(s^{\prime}\right)-N_{\ell}\left(s, s^{\prime}\right) \sum_{I^{\prime \prime \prime}} C_{I^{\prime \prime} I^{\prime \prime \prime}}^{s u} \operatorname{Im} f_{\ell^{\prime}}^{I^{\prime \prime \prime}}\left(s^{\prime}\right)\right]\right\} \\
& +\operatorname{Re} f_{\ell}^{(\text {h.e. }), I}(s) .
\end{aligned}
$$

In equations (6) and (7) the imaginary parts on the right hand side correspond to the so called "input" amplitudes, known in our case from experiment, while the real parts on the left hand side correspond to the "output" from the dispersion relations.

The integrals with the kernels $K, L, N$ and $M$ and high energy parts $R e f_{\ell}^{(h . e .)}(s)$ in Eq. (7) have the same meaning as the kernel and driving terms, respectively, in Roy's equations. Their expressions are lengthy and will be detailed in a future publication. Note that, as the once subtracted GKPY equations have kernel terms that behave as $\sim 1 / s^{2}$ at higher energies, instead of the $\sim 1 / s^{3}$ behavior in Roy's Eqs., the weight of the high energy region is larger. However, as it is seen in Fig. 1 and explained in the next section, it is well under control, as the driving terms are still smaller than the kernel terms. For our purposes here it is enough to describe in detail just the subtraction constant terms in the first line of Eq. (7).

\section{Numerical Results}

Figure 1 presents a decomposition of the equations (6) and (7) into three parts: the subtracting terms $S T(s)$, the kernel terms $K T(s)$ and the driving terms $D T(s)$. This is done for the $S 0, P$ and $S 2$ waves. Note the different scales on the left and right columns in the figure. The numerical calculations have been performed by taking the Constrained Fit to Data amplitudes fitted from experiment in 5 as input. This fit describes the experimental data well, and has been constrained to satisfy Forward Dispersion Relations, Roy's equations and some crossing sum rules. As can be seen in Fig. 1, the $S T(s)$ and $K T(s)$ terms in Roy's Eqs. become huge at higher energies and suffer a strong cancelation. In fact, for a sufficiently large energy, both terms are much larger than the unitarity bound $|R e t| \leq s^{1 / 2} / 2 k \sim 1$, which is only satisfied by the real part of the total amplitude after this strong cancelation. In the case of the $S 2$ and $P$ we do not find such a huge cancelation, since both $S T(s)$ and $K T(s)$ are small enough up to energies of about $s \approx 50 m_{\pi}^{2} \approx(1 \mathrm{GeV})^{2}$.

In the case of the GKPY equations for all waves, however, the $S T(s)$ terms are constant (see eq. 7), and in fact much smaller than the $K T(s)$ terms, which are clearly the dominant ones. Therefore, no big cancellations between any two terms are needed in order to reconstruct the total real part of the amplitude. Note that, although the $D T(s)$ terms in the GKPY equations are larger than in Roy's 
equations due to the fact that there is one subtraction less, they are still small compared with the dominant term $K T(s)$. Thus, the high energy behavior is still well under control.

Figure 2 presents a comparison between the total output amplitudes from Roy's and GKPY equations for the $S 0, P$ and $S 2$ waves. The error bands plotted around the input amplitudes represent the difference between the input and the output, and were generated using a Monte Carlo Gaussian sampling of all parameters in the Constrained Fit to Data (within 6 standard deviations). The asymmetric errors correspond to the independent left and right widths of the generated distribution for $10^{5}$ events. As can be seen on Fig. 2, even though the CFD set of amplitudes was not constrained to fulfill the GKPY equations, they are very well satisfied, with the differences between input and output amplitudes being generally smaller than in the case of Roy's equations. A new Constrained Fit to Data in which the parametrizations are constrained not only to FDR, sum rules and Roy's Eqs., but also to the GKPY equations, and in which the functional form of the parametrizations is refined is in progress.

Especially relevant is that above $s \approx 8 m_{\pi}^{2} \approx(400 \mathrm{MeV})^{2}$ the error bands in all the three waves for the GKPY equations are significantly narrower than those obtained for Roy's equations. As already explained, this comes from the fact that the term $S T(s)$ is a constant, and does not grow with energy, as it was the case with Roy's equations. The errors for the GKPY equations in the three waves come almost completely from the $K T(s)$ terms. As their absolute values are smaller than those of the corresponding functions for Roy's equations, their errors are also smaller above $s \approx 8 m_{\pi}^{2}$. Comparing the non-symmetric widths of the error bands for Roy's equations on Fig. 2 with those calculated in $[5]$ as

$$
\Delta \operatorname{Ret}_{\ell}^{I}=\sqrt{\sum_{j} \delta_{j}^{2}},
$$

where $\delta_{j}$ is the error coming from varying the $j$-th parameter of the CFD set, one obtains, as expected, quite similar results. This is because the errors coming from each individual parameter are small, and the number of parameters is large. In principle the usual Monte Carlo Gaussian sampling keeps a better detail of the correlations, and since they provide asymmetric errors. they will also be used to estimate our errors.

\section{Conclusions}

We have briefly introduced a new set of dispersion relations for $\pi \pi$ partial waves, called GKPY for brevity, with one subtraction and crossing symmetry implemented in a similar way as it is done in Roy Eqs. Both GKPY and Roy Eqs. provide the amplitude as a sum of three kinds of contributions: "subtraction terms" which contain the subtraction constants, "kernel terms" that contain the dispersive integrals of $S 0$-, $P$ - and $S 2$-waves up to a given energy, and the "driving terms" that contain 
contributions from the rest of waves and high energies. As we have shown here, in the case of the new GKPY, the dominant role for the $S 0$-, $P$ - and $S 2$-waves is played by the so called kernel terms. They contain information on the energy dependence of other partial waves below $1420 \mathrm{MeV}$. In contrast, for standard Roy's equations strong cancellations between kernel and subtracting terms occur in the $S 0$ and $S 2$ partial waves, since these terms are several times bigger than the corresponding ones in the GKPY equations. Actually, in Roy Eqs. the subtraction terms grow quadratically with energy and the large experimental uncertainty on the scattering lengths thus propagates to higher energies as a large source of error. Hence, despite Roy Eqs. provide a stringent test for amplitudes at low energy, the GKPY provide an even stronger constraint above roughly $s^{1 / 2}=400 \mathrm{MeV}$, where they have significantly smaller errors than Roy's equations, given the same input. We have also shown here that, although the dependence on the less known high energy input is less suppressed than in standard Roy Eqs., the driving terms are still small in comparison with the KT and ST.

In conclusion, we have shown that GKPY are a good tool to constraint $\pi \pi$ amplitudes in the intermediate energy region. A full data analysis using amplitudes constrained to satisfy simultaneously Forward Dispersion Relations, Roy and GKPY equations is in progress. Once the data analysis is completed, GKPY should also provide a very precise analytic extension to the complex plane that could be relevant for the study of the poles associated to light resonances.

\section{Acknowledgements}

This work is dedicated to memory of our good friend Prof. F. J. Yndurain, who very unfortunately passed away during the completion of this work. We thank Spanish research contracts PR27/05-13955-BSCH, FPA2004-02602, UCM-CAM 910309 and BFM2003-00856 for partial financial support.

\section{References}

1. S.M. Roy, Phys. Lett. B 36, 353 (1971).

2. B. Ananthanarayan et al., Phys. Rept. 353, 207 (2001).

3. G. Colangelo, J. Gasser and H. Leutwyler, Nucl. Phys. B 603, 125 (2001).

4. S. Descotes-Genon, N. H. Fuchs, L. Girlanda and J. Stern, Eur. Phys. J. C 24, 469 (2002)

5. R. Kaminski, J.R. Pelaez and F.J. Yndurain, Phys. Rev. D 77, 054015 (2008).

6. R. Kaminski, J.R. Pelaez and F.J. Yndurain, Phys. Rev. D 74, 014001 (2006). J.R. Pelaez and F.J. Yndurain, Phys. Rev. D 71, 074016 (2005).

7. M. R. Pennington and S. D. Protopopescu, Phys. Rev. D 7, 1429 (1973); Phys. Rev. D 7, 2591 (1973).

8. R. Kaminski, L. Lesniak and B. Loiseau, Phys. Lett. B 551, 241 (2003).

9. S. Pislak et al., Phys. Rev. Lett. 87, 221801 (2001).

10. J. R. Batley et al., CERN-PH-EP-2007-035 (2008).

11. I. Caprini, G. Colangelo, H. Leutwyler, Int. J. Mod. Phys. A 21, 954 (2006); Phys. Rev. Lett. 96, 132001 (2006). 
February 24, 2013 15:12 WSPC/INSTRUCTION FILE RKaminskiMeson2008

New dispersion relations in the description of $\pi \pi$ scattering amplitudes 7
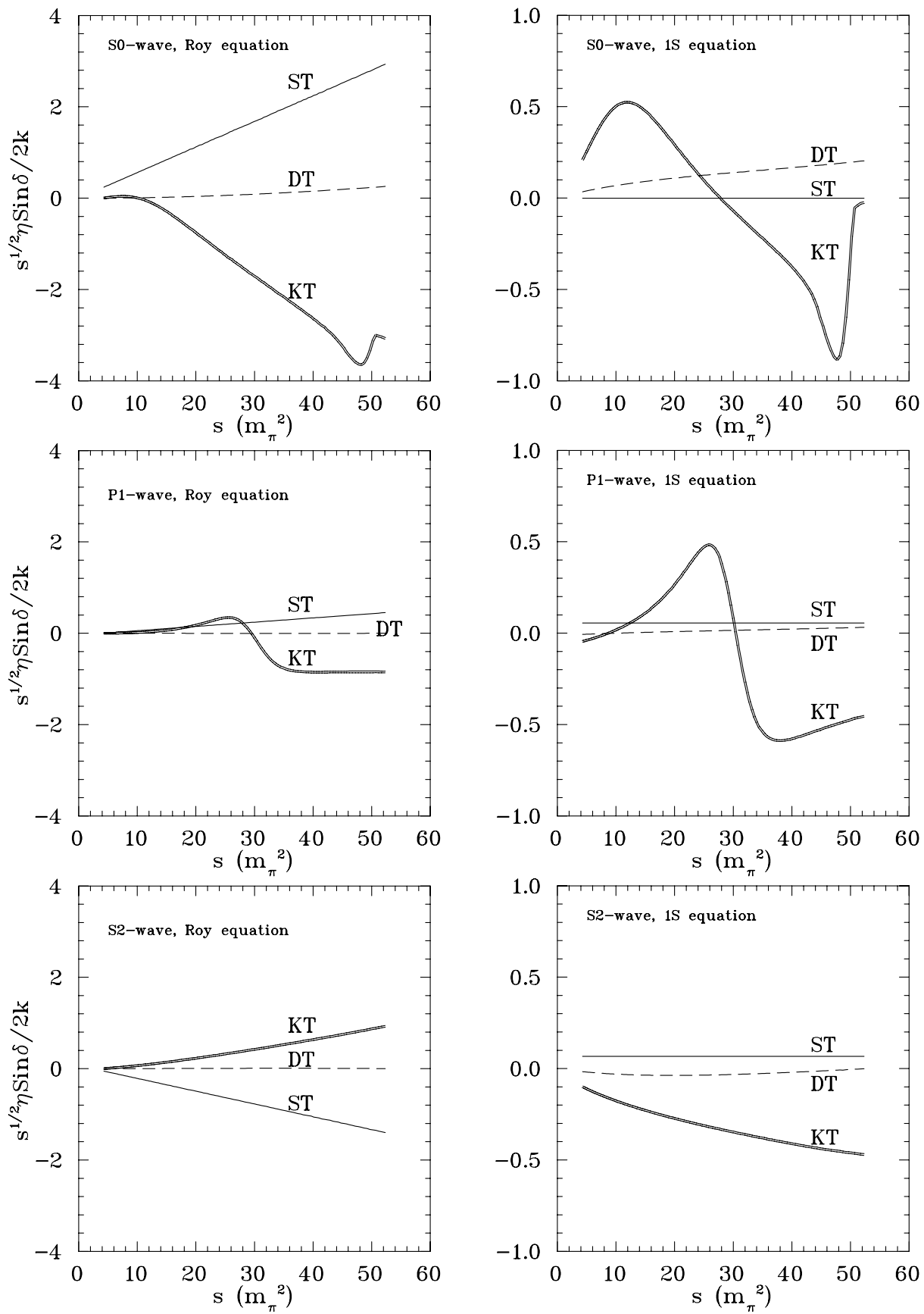

Fig. 1. Decomposition of the results from Roy's and $1 \mathrm{~S}$ equations into subtracting term ST, kernel term KT, and driving term DT for the $S 0$-, $P$ - and $S 2$-waves. Note the different scales used in the left and right columns. 

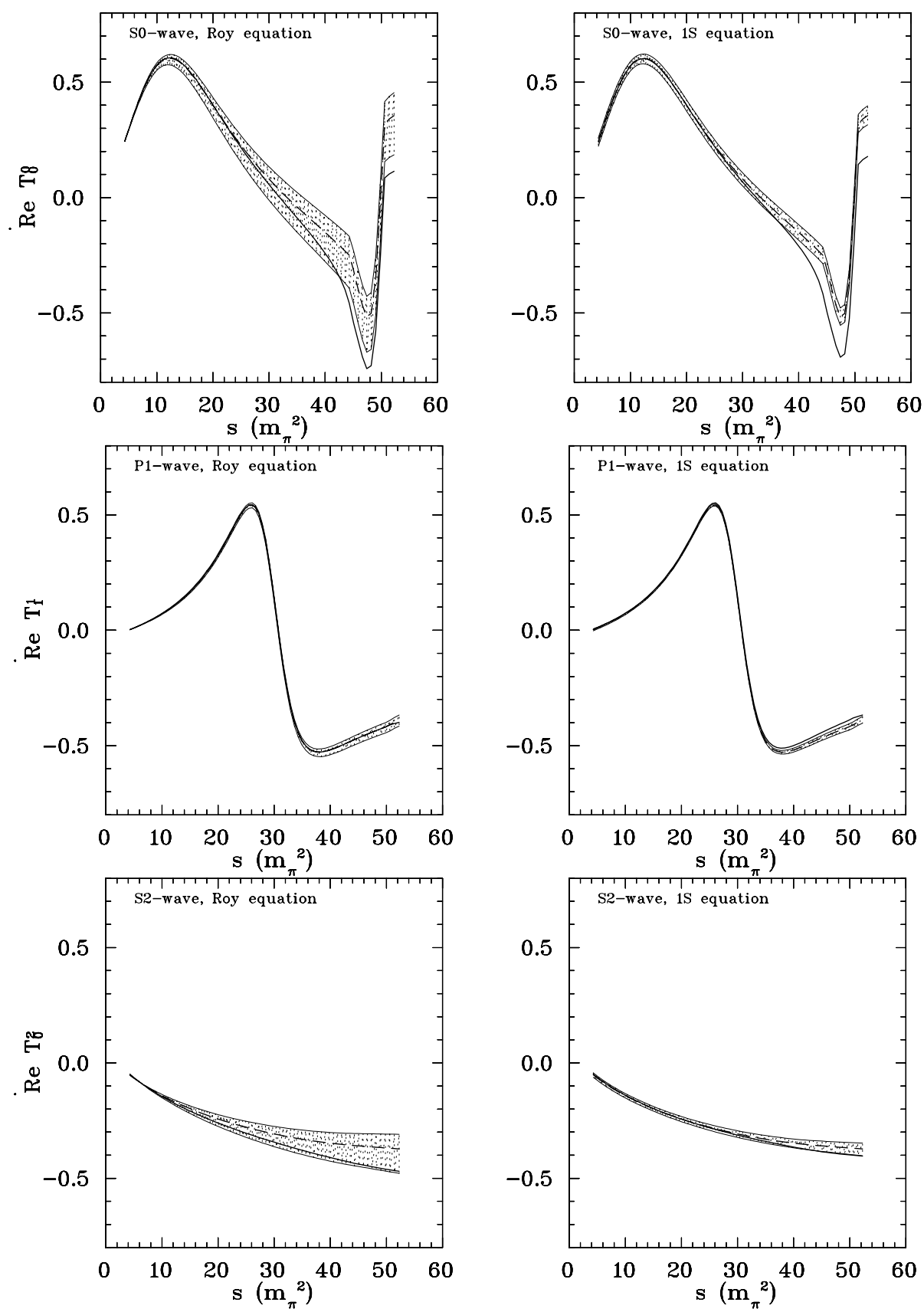

Fig. 2. Comparison of results from Roy's and $1 \mathrm{~S}$ equations for waves $S 0, P$ and $S 2$. The gray bands correspond to the errors for these equations. The dashed and solid lines represent the input and output amplitudes, respectively. 